\title{
Effect of Food and Temperature on The Biology of Typhlodrompis swirskii (Athias-Henriot) (Acari: Phytoseiidae)
}

\author{
Fatma S. Ali and M. A. Zaher \\ Zoology and Agric. Nematology Dept., Faculty of Agriculture, Cairo University, Egypt
}

\begin{abstract}
The biology of Typhlodrompis swirskii (Athias-Henriot) fed on different associated phytophagous mites, insects and pollens was studied at $25^{\circ}$ C. These were Eutetranychus orientalis (Klien) (immatures and adults), Cenopalpus pulcher (C. \& F.) females, Panonychus ulmi (Koch) (females), Tetranychus urticae Koch (immatures and eggs), Tydeus californicus (Banks) moving stages; whitefly Bemisia tabaci (Gennadius) (eggs and $1^{\text {st }} \& 2^{\text {nd }}$ instar nymphs), the scale insects Chrysomphalus ficus Rilly and Coccus hesperidium (Linnaeus) (eggs and nymphs), the aphid Aphis duranta Theo (adults), date palm pollens Phoenix dactylifera L. and castor bean pollens Ricinus communis L. Amount of consumption, as well as the predation capacity were estimated. Mites and date palm pollens were more suitable than insects and castor bean pollens; also immatures of $E$. orientalis gave the shortest female life cycle (10.8 days) and the highest female fecundity (38.0 eggs/o). Feeding T. swirskii on E. orientalis immatures at 15, 20, 25, 28, 30, 32 and $35^{\circ} \mathrm{C}$, the developmental durations averaged 18.5, 14.3, 10.8, 9.0, 7.7, 6.8 and 6.1 days respectively. The intrinsic rate of natural increase (rm) and the net reproduction (Ro) represented the best values 0.269 and 26.48 respectively at $32^{\circ} \mathrm{C}$ the mean generation time decreased with increasing temperature $\left(10.971\right.$ days at $\left.35^{\circ} \mathrm{C}\right)$.
\end{abstract}

KEY WORDS: Typhlodrompis swirskii, Phytoseiidae, biology, different prey, temperature.

\section{INTRODUCTION}

Plant inhabiting predaceous mites play a considerable role in the biological control of associated pests, such as phytophagous mites, scale insects and whiteflies. Members of the family Phytoseiidae proved to be the most important due to their high predaceous efficiency, world wide distribution and large number of species which exceeds 1700 species (Walter 1992). Some are important in controlling phytophagous mites and insects, of which certain species such as Phytoseiulus persimilis (Athias-Henriot) Neoseiulus (=Amblyseius) californicus (McG.) Metaseiulus (=Typhlodromus) occidentalis (Nesbitt) and Phytoseiulus macropilis (Banks) have already bean used as biocontrol agents of the spider mites in greenhouses and open fields (El-laithy, 1992; Decou, 1994, Watanabe et al., 1994; Remos \& Rodriguez, 1995; Ali, 1998; Heikal \& Mowafi, 1998; Heikal et al., 2000 and Heikal \& Ibrahim, 2001).

Typhlodrompis swirskii (Athias-Henriot) is a common phytoseiid mite on fruit trees in Egypt, (Zaher, 1986) Therefore, the present study aimed to get more information on some biological and ecological aspects to clear out its importance as a biocontrol agent for some insect and mite pests.

\section{MATERIALS AND METHODS}

T. swirskii was found on leaves of grape fruit, Citrus paradisi (Macfad), apple, Malus sylvestris Mill, and guava, Psidium guajava L., orchards in
Behaira, Ismailia, Monofia and Gharbia governorates, Egypt. Culture of this species was established at Acarology Laboratory, of the Faculty of Agriculture, Cairo University.

\section{Rearing of predacious mite:}

For solitary rearing, newly deposited eggs were transferred singly each to a mango leaf disc of one square inch. Each newly hatched larva was supplied with a known number of prey and devoured ones were replaced daily by fresh ones till reaching maturity. Emerged females were copulated for oviposition. Large plastic boxes $25 \times 15 \times 5 \mathrm{~cm}$ were used. A cotton pad was put on the bottom of each box, leaving a space of about one $\mathrm{cm}$ provided with water as a barrier to prevent predatory mite individuals from escaping, in addition to a tangle foot strip at the box edges. About 10 leaf discs with solitary predator larvae were placed on the cotton pad of every box. Excised bean leaves with a known number of the citrus brown mite, Eutetranychus orientalis (Klein) immatures was provided as food sources. Plastic boxes were kept in an incubator at $25 \pm 1{ }^{\circ} \mathrm{C}$ and $70 \pm 5 \%$ R.H. Each experiment was started with at least 25 newly hatched predator larvae and observations were recorded every $12 \mathrm{~h}$.

\section{Food sources:}

Immatures and adults of E. orientalis; immatures and eggs of Tetranychus urticae Koch; adults of Panonychus ulmi (Koch) (Tetranychidae); adults of Cenopalpus pulcher (C. \& F.) (Tenuipalpidae); moving stages of Cisaberoptus kenyae Keifer (Eriophyidae); \& of Tydeus californicus (Banks) (Tydeidae); eggs and $1^{\text {st }}, 2^{\text {nd }}$ instar nymphs of the whitefly Bemisia tabaci (Gennadius); eggs and 
nymphs of the scale insects Chrysomphalus ficus Rilly and Coccus hesperidium (Linnaeus); adults of the aphid Aphis duranta Theo; date palm pollens Phoenix dactylifera L. and castor bean pollens Ricinus communis L. were used as food. For testing food consumption, known numbers of every prey were offered to each predator individual, and devoured prey were replaced with fresh ones daily.

\section{Life tables:}

An experiment was conducted for the influence of temperature on the life table parameters of T. swirskii using the most suitable prey (E. orientalis, immatures) in incubators at different temperatures $\left(15,20,25,28,30,32\right.$ and $\left.35^{\circ} \mathrm{C}\right)$ and calculated according to the life 48 computer program (Abou-Setta et al., 1986).

\section{RESULTS AND DISCUSSION}

T. swirskii is considered a predator of certain phytophagous mites and insects. Field observations showed that this mite usually inhabits the lower leaf surface preferring the area between veins. Eggs of this mite were translucent and when females fed on the tenuipalpid mite $C$. pulcher it appeared pale reddish, while the tetranychids (Tetranychus, \& Eutetra-nychus) gave a yellowish appearance and date palm resulted also in yellowish or damp whitish colour.

A. swirskii was reared on different associated phytophagous mites and insects as well as pollens. These were E. orientalis (immatures and adults), C. pulcher (females), P. ulmi (females), T. urticae (immatures and eggs), C. kenyae \& T. californicus (moving stages); the scale insects $C$. ficus (nymphs and eggs) and $C$. hesperidium (nymphs and eggs), the whitefly B. tabaci $\left(2^{\text {nd }}\right.$ and $1^{\text {st }}$ instar nymphs and eggs), the aphid A. duranta (adults); and date palm and castor bean pollens at $25^{\circ} \mathrm{C}$. On the opposite, rearing was unsuccessful on $T$. californicus and castor bean pollens. (Table1). Mites and date palm pollens were more suitable than insects and castor bean pollens; also immatures were better than eggs and adults. At $25^{\circ} \mathrm{C}$, E. orientalis immatures favoured the shortest duration of predator life cycle (10.8 days) and adult longevity (37.4 days) and highest fecundity (38.0 eggs/O) while $C$. kenyae gave the longest durations ( $20.0 \& 77.4$ days) and the lowest fecundity ( $8.8 \mathrm{eggs} /$ 9 ). The intrinsic rate of natural increase (rm) amounted 0.161 and 0.027 individual/ $9 /$ day for the two prey, respectively. The net reproductive rate (Ro) was the highest on $E$. orientalis (16.39 times), while being the lowest (2.88 times) on C. kenyae. T. swirskii had a wide range of hosts, but usually found on citrus, thus preferring $E$. orientalis than other tested mites. It also preferred immatures than adults, for its smaller size and soft skin. These results support the findings of van de Vrie \& Kropczynska (1965) and van de Vrie and Boersma (1970) for Typhlodromus pyri Scheuten and Typhlodrompis potentillae (German) and Caceres \& Childers (1991) for Galendromus helveolus (Chant) for preferring immatures of spider mites. They attributed this to the large size of adult prey or its ability to spin webs. Table (2) showed that $T$. swirskii fed on insects of which $B$. tabaci $2^{\text {nd }}$ instar nymphs gave the shortest life cycle and adult longevity (15.3 \& 60.3 days) and the highest fecundity (18.5 eggs). A. duranta adults gave the longest periods ( $19.4 \& 75.2$ days) and the lowest fecundity (9.4 eggs), respectively. Male predator life span followed similar trend except having shorter periods. On the contrary, rearing on T. californicus and castor bean pollens, the predator could not complete its development. The intrinsic rate of natural increase $(\mathrm{rm})$ was in a descending order $0.063,0.061,0.056,0.047,0.042,0.036,0.034$ and 0.027 individual/o $/$ day on $B$. tabaci $2^{\text {nd }}, 1^{\text {st }}$ instar nymphs and eggs, $C$. ficus and $C$. hesperidium nymphs \& eggs and A. duranta adults, (Table 2).

Table (1): Duration of developmental stages and life table parameters of Typhlodrompis swirskii fed on mites and pollens at $25^{\circ} \mathrm{C}$.

\begin{tabular}{|c|c|c|c|c|c|c|c|c|c|c|}
\hline & \multicolumn{9}{|c|}{ Food } \\
\hline & & \multicolumn{2}{|c|}{ E. orientalis } & \multirow{2}{*}{$\begin{array}{l}\text { Date palm } \\
\text { pollens }\end{array}$} & \multirow{2}{*}{$\begin{array}{l}\text { C. pulcher } \\
\text { Adults }\end{array}$} & \multirow{2}{*}{$\begin{array}{l}P \text {. ulmi } \\
\text { Adults }\end{array}$} & \multicolumn{2}{|c|}{ T. urticae } & \multirow{2}{*}{$\begin{array}{c}C . \\
\text { kenyae }\end{array}$} & \multirow{2}{*}{$\begin{array}{c}T . \\
\text { californicus }\end{array}$} \\
\hline & & Immatures & Adults & & & & Immatures & Eggs & & \\
\hline \multirow{2}{*}{ Life cycle } & q & $10.8 \pm 1.09$ & $11.3 \pm 0.52$ & $12.3 \pm 1.37$ & $13.2 \pm 0.83$ & $13.8 \pm 1.48$ & $14.2 \pm 0.75$ & $14.7 \pm 0.52$ & $20.0 \pm 0.70$ & $21.4 \pm 0.89$ \\
\hline & $0^{1}$ & $10.2 \pm 1.30$ & $10.8 \pm 0.98$ & $11.7 \pm 0.82$ & $12.8 \pm 1.30$ & $13.2 \pm 1.30$ & $13.5 \pm 0.83$ & $14.0 \pm 1.09$ & $19.0 \pm 1.41$ & $20.8 \pm 0.83$ \\
\hline \multirow{2}{*}{$\begin{array}{l}\text { Adult } \\
\text { longevity }\end{array}$} & 오 & $37.4 \pm 1.14$ & $40.2 \pm 1.16$ & $43.8 \pm 1.60$ & $48.6 \pm 0.89$ & $50.8 \pm 1.30$ & $53.3 \pm 1.37$ & $58.5 \pm 1.04$ & $77.4 \pm 1.67$ & -- \\
\hline & $0^{\pi}$ & $30.0 \pm 0.70$ & $33.2 \pm 0.75$ & $36.2 \pm 0.75$ & $40.2 \pm 0.83$ & $44.2 \pm 0.83$ & $47.2 \pm 1.16$ & $52.2 \pm 0.75$ & $69.8 \pm 0.83$ & -- \\
\hline \multicolumn{2}{|c|}{ Total ggs/female } & $38.0 \pm 1.00$ & $34.2 \pm 0.75$ & $30.5 \pm 0.54$ & $28.2 \pm 0.83$ & $25.2 \pm 0.83$ & $22.8 \pm 0.75$ & $21.0 \pm 0.89$ & $8.8 \pm 0.83$ & -- \\
\hline \multicolumn{2}{|l|}{ Eggs daily rate } & $1.30 \pm 0.03$ & $1.12 \pm 0.04$ & $0.94 \pm 0.08$ & $0.82 \pm 0.03$ & $0.70 \pm 0.03$ & $0.61 \pm 0.04$ & $0.53 \pm 0.00$ & $0.83 \pm 0.01$ & -- \\
\hline \multicolumn{2}{|l|}{ Ro } & 16.39 & 17.65 & 16.11 & 11.43 & 10.14 & 7.40 & 6.92 & 2.88 & -- \\
\hline \multicolumn{2}{|c|}{$\mathrm{T}$} & 17.28 & 18.49 & 21.45 & 22.94 & 23.60 & 25.92 & 25.52 & 38.72 & -- \\
\hline \multicolumn{2}{|l|}{$\mathrm{rm}$} & 0.161 & 0.155 & 0.129 & 0.106 & 0.098 & 0.077 & 0.075 & 0.027 & -- \\
\hline \multirow{2}{*}{\multicolumn{2}{|c|}{$\exp \operatorname{rm}(\lambda)$}} & 1.175 & 1.167 & 1.138 & 1.112 & 1.103 & 1.080 & 1.078 & 1.027 & -- \\
\hline & & 0.76 & 0.75 & 0.75 & 0.71 & 0.69 & 0.69 & 0.69 & 0.64 & -- \\
\hline
\end{tabular}


Table (2): Duration of developmental stages and life table parameters of Typhlodrompis swirskii fed on insects at $25^{\circ} \mathrm{C}$.

\begin{tabular}{|c|c|c|c|c|c|c|c|c|c|}
\hline & \multicolumn{8}{|c|}{ Food } \\
\hline & & \multicolumn{3}{|c|}{ B. tabaci } & \multicolumn{2}{|c|}{ Ch. ficus } & \multicolumn{2}{|c|}{ C. hesperdium } & \multirow{2}{*}{$\frac{\text { A. duranta }}{\text { Adults }}$} \\
\hline & & $2^{\text {nd }}$ nymphs & $1^{\text {st }}$ nymphs & Eggs & Nymphs & Eggs & Nymphs & Eggs & \\
\hline \multirow{2}{*}{ Life cycle } & 오 & $15.3 \pm 1.21$ & $15.8 \pm 1.16$ & $16.3 \pm 1.03$ & $17.0 \pm 0.70$ & $18.0 \pm 0.70$ & $18.5 \pm 1.37$ & $19.0 \pm 1.26$ & $19.4 \pm 0.89$ \\
\hline & $\hat{0}$ & $14.6 \pm 1.51$ & $15.2 \pm 1.30$ & $15.5 \pm 1.37$ & $16.2 \pm 1.30$ & $17.2 \pm 0.44$ & $17.7 \pm 1.21$ & $18.2 \pm 1.32$ & $18.4 \pm 1.51$ \\
\hline \multirow{2}{*}{$\begin{array}{l}\text { Adult } \\
\text { longevity }\end{array}$} & 오 & $60.3 \pm 0.81$ & $63.7 \pm 1.96$ & $65.8 \pm 1.16$ & $68.2 \pm 1.64$ & $71.6 \pm 1.15$ & $72.5 \pm 1.37$ & $73.5 \pm 1.22$ & $75.2 \pm 1.64$ \\
\hline & $\hat{0}$ & $54.2 \pm 0.83$ & $56.0 \pm 0.70$ & $57.0 \pm 0.89$ & $63.0 \pm 1.00$ & $65.2 \pm 0.83$ & $65.7 \pm 0.81$ & $67.7 \pm 0.81$ & $68.8 \pm 0.83$ \\
\hline \multicolumn{2}{|c|}{ Total ggs/female } & $18.5 \pm 1.04$ & $18.0 \pm 0.89$ & $16.0 \pm 0.89$ & $14.0 \pm 0.70$ & $12.0 \pm 0.70$ & $11.0 \pm 0.89$ & $10.5 \pm 0.54$ & $9.4 \pm 0.54$ \\
\hline \multicolumn{2}{|c|}{ Eggs daily rate } & $0.46 \pm 0.02$ & $0.43 \pm 0.02$ & $0.37 \pm 0.01$ & $0.31 \pm 0.01$ & $0.26 \pm 0.02$ & $0.23 \pm 0.01$ & $0.22 \pm 0.01$ & $0.20 \pm 0.01$ \\
\hline \multicolumn{2}{|l|}{ Ro } & 5.77 & 5.68 & 5.47 & 4.53 & 3.96 & 3.57 & 3.45 & 2.82 \\
\hline \multicolumn{2}{|l|}{$\mathrm{T}$} & 27.50 & 28.16 & 29.87 & 31.76 & 32.59 & 34.83 & 35.71 & 37.10 \\
\hline \multicolumn{2}{|l|}{$\mathrm{rm}$} & 0.063 & 0.061 & 0.056 & 0.047 & 0.042 & 0.036 & 0.034 & 0.027 \\
\hline \multicolumn{2}{|l|}{$\exp \operatorname{rm}(\lambda)$} & 1.065 & 1.063 & 1.058 & 1.048 & 1.043 & 1.037 & 1.035 & 1.020 \\
\hline \multicolumn{2}{|c|}{ Sex ratio $(90 \%)$} & 0.69 & 0.67 & 0.67 & 0.66 & 0.66 & 0.64 & 0.64 & 0.64 \\
\hline
\end{tabular}

Table (3): Duration of developmental stages and life table parameters of Typhlodrompis swirskii fed on Eutetranychus orientalis immatures at different temperatures

\begin{tabular}{|c|c|c|c|c|c|c|c|c|}
\hline & \multicolumn{7}{|c|}{ Temperature ${ }^{\circ} \mathrm{C}$} \\
\hline & & 15 & 20 & 25 & 28 & 30 & 32 & 35 \\
\hline \multirow{2}{*}{ Life cycle } & 웅 & $18.5 \pm 0.54$ & $14.3 \pm 0.82$ & $10.8 \pm 1.09$ & $9.0 \pm 1.22$ & $7.7 \pm 0.52$ & $6.8 \pm 0.70$ & $6.1 \pm 1.02$ \\
\hline & i & $17.4 \pm 0.89$ & $13.8 \pm 0.75$ & $10.2 \pm 1.30$ & $8.2 \pm 1.09$ & $7.2 \pm 0.83$ & $6.2 \pm 0.44$ & $5.7 \pm 0.44$ \\
\hline \multirow{2}{*}{$\begin{array}{l}\text { Adult } \\
\text { longevity }\end{array}$} & 오 & $60.3 \pm 0.21$ & $50.8 \pm 0.78$ & $37.4 \pm 1.14$ & $32.6 \pm 0.89$ & $29.7 \pm 1.37$ & $26.2 \pm 1.32$ & $23.5 \pm 1.04$ \\
\hline & $\hat{0}$ & $51.0 \pm 1.22$ & $41.7 \pm 1.37$ & $30.0 \pm 0.70$ & $26.4 \pm 0.54$ & $24.2 \pm 0.83$ & $20.8 \pm 0.44$ & $17.4 \pm 0.89$ \\
\hline \multicolumn{2}{|c|}{ Total ggs/female } & $18.3 \pm 0.82$ & $22.0 \pm 0.89$ & $38.0 \pm 1.00$ & $41.8 \pm 0.83$ & $45.7 \pm 0.82$ & $47.8 \pm 0.75$ & $48.7 \pm 0.52$ \\
\hline \multicolumn{2}{|c|}{ Eggs daily rate } & $0.49 \pm 0.01$ & $0.64 \pm 0.01$ & $1.30 \pm 0.03$ & $1.60 \pm 0.05$ & $1.90 \pm 0.06$ & $2.20 \pm 0.13$ & $2.40 \pm 0.04$ \\
\hline \multicolumn{2}{|l|}{ Ro } & 7.738 & 10.950 & 16.392 & 26.116 & 23.094 & 26.486 & 24.064 \\
\hline \multicolumn{2}{|c|}{$\mathrm{T}$} & 29.606 & 23.452 & 17.283 & 16.104 & 13.805 & 12.145 & 10.971 \\
\hline \multicolumn{2}{|l|}{$\mathrm{rm}$} & 0.069 & 0.102 & 0.161 & 0.202 & 0.227 & 0.269 & 0.289 \\
\hline \multicolumn{2}{|c|}{$\operatorname{exprm}(\lambda)$} & 1.071 & 1.107 & 1.175 & 1.224 & 1.255 & 1.309 & 1.336 \\
\hline \multicolumn{2}{|c|}{ Sex ratio (OQ\%) } & 0.71 & 0.73 & 0.76 & 0.76 & 0.76 & 0.76 & 0.78 \\
\hline
\end{tabular}

Table (4): Food consumption of Typhlodrompis swirskii on different stages of tetranychoid mites during its life span at $25^{\circ} \mathrm{C}$.

\begin{tabular}{|c|c|c|c|c|c|c|c|c|c|c|c|c|c|}
\hline \multirow{4}{*}{ Predator } & & \multicolumn{12}{|c|}{ No. of devoured prey } \\
\hline & & \multicolumn{4}{|c|}{ E. orientalis } & \multirow{2}{*}{\multicolumn{2}{|c|}{$\begin{array}{c}\text { C. pulcher } \\
\text { Adults }\end{array}$}} & \multirow{2}{*}{\multicolumn{2}{|c|}{$\begin{array}{l}\text { P. ulmi } \\
\text { Adults }\end{array}$}} & \multicolumn{4}{|c|}{ T. urticae } \\
\hline & & \multicolumn{2}{|c|}{ Immatures } & \multicolumn{2}{|l|}{ Adults } & & & & & \multicolumn{2}{|c|}{ Immatures } & \multicolumn{2}{|l|}{ Eggs } \\
\hline & & $\mathrm{T}$ & D.R & $\mathrm{T}$ & D.R & $\mathrm{T}$ & D.R & $\mathrm{T}$ & D.R & $\mathrm{T}$ & D.R & $\mathrm{T}$ & D.R \\
\hline \multirow{2}{*}{$\begin{array}{l}\text { Total } \\
\text { immatures }\end{array}$} & $q$ & $21.3 \pm 1.21$ & 3.7 & $17.2 \pm 1.83$ & 3.1 & $14.2 \pm 0.75$ & 2.4 & $13.2 \pm 0.40$ & 2.2 & $10.3 \pm 1.03$ & 1.9 & $9.8 \pm 0.75$ & 1.8 \\
\hline & $\hat{\sigma}$ & $15.2 \pm 0.83$ & 3.1 & $12.2 \pm 1.30$ & 2.6 & $10.2 \pm 0.83$ & 2.0 & $9.0 \pm 1.00$ & 1.9 & $7.2 \pm 0.44$ & 1.7 & $6.4 \pm 1.14$ & 1.6 \\
\hline Oviposition & & $119.0 \pm 1.41$ & 4.1 & $114.7 \pm 2.44$ & 3.8 & $112.3 \pm 1.36$ & 3.3 & $108.7 \pm 1.21$ & 3.0 & $102.7 \pm 1.96$ & 2.8 & $99.5 \pm 1.51$ & 2.6 \\
\hline \multirow{2}{*}{$\begin{array}{l}\text { Adult } \\
\text { longevity }\end{array}$} & q & $148.0 \pm 1.67$ & 4.0 & $140.3 \pm 2.94$ & 3.5 & $136.5 \pm 1.22$ & 2.8 & $129.7 \pm 0.98$ & 2.6 & $120.7 \pm 1.50$ & 2.3 & $116.2 \pm 1.72$ & 2.0 \\
\hline & $\hat{0}$ & $115.4 \pm 2.30$ & 3.8 & $11.4 \pm 1.81$ & 3.3 & $107.6 \pm 1.51$ & 2.7 & $99.4 \pm 1.14$ & 2.2 & $93.2 \pm 1.92$ & 2.0 & $88.8 \pm 1.30$ & 1.7 \\
\hline \multirow{2}{*}{ Life span } & 운 & $169.3 \pm 2.50$ & 3.5 & $157.5 \pm 4.32$ & 3.1 & $150.7 \pm 1.21$ & 2.4 & $142.8 \pm 0.75$ & 2.2 & $131.0 \pm 1.89$ & 1.9 & $126.0 \pm 2.44$ & 1.7 \\
\hline & $\hat{0}$ & $130.6 \pm 2.07$ & 3.2 & $123.6 \pm 2.19$ & 2.8 & $117.8 \pm 1.48$ & 2.2 & $108.4 \pm 2.07$ & 1.9 & $100.4 \pm 1.51$ & 1.7 & $95.2 \pm 1.48$ & 1.4 \\
\hline
\end{tabular}

$$
\text { T. }=\text { Total D.R. = Daily Rate }
$$


Table (5): Food consumption of Typhlodrompis swirskii on different stages of insects during life span at $25^{\circ} \mathrm{C}$

\begin{tabular}{|c|c|c|c|c|c|c|c|c|c|c|c|c|c|c|c|c|c|}
\hline \multirow{4}{*}{ Predator } & & \multicolumn{16}{|c|}{ No. of devoured prey } \\
\hline & & \multicolumn{6}{|c|}{ B. tabaci } & \multicolumn{4}{|c|}{ Ch. ficus } & \multicolumn{4}{|c|}{ C. hesperidium } & \multirow{2}{*}{\multicolumn{2}{|c|}{ A. duranta }} \\
\hline & & \multicolumn{2}{|c|}{$2^{\text {nd }}$ instar nymphs } & \multicolumn{2}{|l|}{$\begin{array}{l}1^{\text {st }} \text { instar } \\
\text { nymphs }\end{array}$} & \multicolumn{2}{|l|}{ Eggs } & \multicolumn{2}{|l|}{ Nymphs } & \multicolumn{2}{|c|}{$\begin{array}{l}2^{\text {nd }} \text { instar } \\
\text { mymphs }\end{array}$} & \multicolumn{2}{|c|}{$\begin{array}{l}1^{\text {st }} \text { instar } \\
\text { nymphs }\end{array}$} & \multicolumn{2}{|l|}{ Eggs } & & \\
\hline & & $\mathrm{T}$ & D.R & $\mathrm{T}$ & D.R & $\mathrm{T}$ & D.R & $\mathrm{T}$ & D.R & $\mathrm{T}$ & D.R & $\mathrm{T}$ & D.R & $\mathrm{T}$ & D.R & $\mathrm{T}$ & D.R \\
\hline \multirow{2}{*}{$\begin{array}{l}\text { Total } \\
\text { immatures }\end{array}$} & 운 & $8.8 \pm 0.98$ & 1.7 & $8.2 \pm 1.16$ & 1.6 & $7.0 \pm 0.89$ & 1.5 & $6.2 \pm 0.40$ & 1.4 & $5.5 \pm 0.83$ & 1.4 & $4.7 \pm 0.83$ & 1.3 & $4.3 \pm 0.51$ & 1.3 & $3.7 \pm 0.51$ & 1.2 \\
\hline & $\hat{0}$ & $4.5 \pm 0.54$ & 1.5 & $4.2 \pm 1.30$ & 1.3 & $3.4 \pm 0.89$ & 1.3 & $2.6 \pm 0.54$ & 1.2 & $2.2 \pm 0.83$ & 1.1 & $1.8 \pm 1.09$ & 1.1 & $1.4 \pm 0.89$ & 1.1 & $1.0 \pm 0.00$ & 1.0 \\
\hline Oviposition & & $97.0 \pm 0.89$ & 2.4 & $93.7 \pm 2.16$ & 2.3 & $88.3 \pm 1.50$ & 2.1 & $85.8 \pm 0.75$ & 2.0 & $81.0 \pm 1.26$ & 1.8 & $77.3 \pm 1.21$ & 1.7 & $73.7 \pm 1.21$ & 1.7 & $61.8 \pm 1.60$ & 1.3 \\
\hline \multirow{2}{*}{$\begin{array}{l}\text { Adult } \\
\text { longevity }\end{array}$} & q & $112.3 \pm 1.50$ & 1.9 & $108.0 \pm 1.78$ & 1.7 & $101.0 \pm 2.00$ & 1.5 & $98.5 \pm 1.04$ & 1.5 & $92.3 \pm 1.32$ & 1.5 & $88.0 \pm 1.41$ & 1.4 & $83.7 \pm 1.04$ & 1.2 & $70.0 \pm 1.09$ & 1.2 \\
\hline & $\delta$ & $83.0 \pm 1.87$ & 1.5 & $81.2 \pm 0.83$ & 1.4 & $78.0 \pm 1.85$ & 1.4 & $75.8 \pm 0.83$ & 1.2 & $72.4 \pm 1.51$ & 1.1 & $69.6 \pm 1.14$ & 1.2 & $66.8 \pm 1.78$ & 1.0 & $52.6 \pm 1.67$ & 1.0 \\
\hline Life span & q & $121.2 \pm 0.98$ & 1.6 & $116.2 \pm 2.56$ & 1.5 & $108.0 \pm 2.0$ & 1.3 & $104.7 \pm 0.81$ & 1.2 & $97.8 \pm 1.75$ & 1.1 & $92.7 \pm 2.06$ & 1.0 & $88.0 \pm 1.32$ & 0.9 & $73.7 \pm 1.21$ & 0.6 \\
\hline
\end{tabular}

Table (6): Food consumption of Typhlodrompis swirskii on E. orientalis immatures at different temperatures during its life span

\begin{tabular}{|c|c|c|c|c|c|c|c|c|c|c|c|c|c|c|c|}
\hline & & \multicolumn{14}{|c|}{ No. of devoured prey immatures } \\
\hline & & \multicolumn{2}{|l|}{$15^{\circ} \mathrm{C}$} & \multicolumn{2}{|l|}{$20^{\circ} \mathrm{C}$} & \multicolumn{2}{|l|}{$25^{\circ} \mathrm{C}$} & \multicolumn{2}{|l|}{$28^{\circ} \mathrm{C}$} & \multicolumn{2}{|l|}{$30^{\circ} \mathrm{C}$} & \multicolumn{2}{|l|}{$32^{\circ} \mathrm{C}$} & \multicolumn{2}{|l|}{$35^{\circ} \mathrm{C}$} \\
\hline & & $\mathrm{T}$ & D.R & $\mathrm{T}$ & D.R & $\mathrm{T}$ & D.R & $\mathrm{T}$ & D.R & $\mathrm{T}$ & D.R & $T$ & D.R & $\mathrm{T}$ & D.R \\
\hline \multirow{2}{*}{$\begin{array}{l}\text { Total } \\
\text { immatures }\end{array}$} & q & $5.0 \pm 1.26$ & 1.4 & $7.0 \pm 1.89$ & 1.6 & $21.3 \pm 1.21$ & 3.7 & $24.7 \pm 1.16$ & 4.5 & $28.3 \pm 2.58$ & 5.9 & $34.0 \pm 1.67$ & 7.1 & $37.3 \pm 1.50$ & 12.9 \\
\hline & $\hat{0}$ & $4.2 \pm 1.30$ & 1.3 & $6.0 \pm 1.22$ & 1.6 & $15.2 \pm 0.83$ & 3.1 & $17.4 \pm 1.00$ & 3.8 & $19.4 \pm 1.14$ & 4.7 & $21.8 \pm 1.14$ & 5.2 & $25.2 \pm 1.09$ & 6.8 \\
\hline Oviposition & & $13.8 \pm 0.75$ & 1.3 & $16.0 \pm 0.63$ & 1.5 & $119.0 \pm 0.89$ & 4.1 & $129.5 \pm 0.75$ & 5.4 & $138.2 \pm 0.89$ & 6.5 & $144.0 \pm 0.81$ & 9.5 & $150.2 \pm 1.03$ & 10.5 \\
\hline \multirow{2}{*}{$\begin{array}{l}\text { Adult } \\
\text { longevity }\end{array}$} & 울 & $23.7 \pm 1.86$ & 1.5 & $29.5 \pm 1.87$ & 1.7 & $148.0 \pm 1.67$ & 4.6 & $164.0 \pm 1.26$ & 3.6 & $176.5 \pm 1.67$ & 6.9 & $185.3 \pm 3.20$ & 9.9 & $194.5 \pm 2.42$ & 13.0 \\
\hline & $\hat{0}$ & $13.6 \pm 1.14$ & 1.4 & $18.2 \pm 0.83$ & 1.4 & $115.4 \pm 2.30$ & 3.8 & $125.6 \pm 3.50$ & 4.7 & $134.6 \pm 2.96$ & 6.2 & $139.0 \pm 2.42$ & 6.7 & $140.8 \pm 0.83$ & 8.1 \\
\hline \multirow{2}{*}{ Life span } & q & $28.7 \pm 1.50$ & 1.5 & $36.5 \pm 1.51$ & 1.6 & $169.3 \pm 2.50$ & 4.5 & $188.7 \pm 1.03$ & 5.5 & $125.3 \pm 2.87$ & 6.5 & $219.3 \pm 4.27$ & 9.7 & $231.8 \pm 2.78$ & 10.8 \\
\hline & $\hat{0}$ & $17.8 \pm 2.16$ & 0.3 & $24.2 \pm 1.78$ & 0.4 & $130.6 \pm 2.07$ & 3.2 & $143.0 \pm 2.44$ & 4.1 & $154.0 \pm 2.91$ & 4.9 & $160.8 \pm 2.28$ & 5.9 & $166.0 \pm 1.20$ & 7.2 \\
\hline
\end{tabular}

The effect of temperatures on life cycle, adult longevity and female fecundity of $T$. swirskii fed on $E$. orientalis immatures which proved to be the most suitable food was shown in table (3). As temperature increased from 15 to $35^{\circ} \mathrm{C}$, the female life cycle duration as well as adult longevity and fecundity ranged from $18.5 \& 60.3$ days and 18.3 eggs to 6.1 $\& 23.5$ days and 48.7 eggs. The intrinsic rate of increase (rm) increased with increasing temperature from 15 to $35^{\circ} \mathrm{C}$; this value ranged from 0.069 to 0.289 individuals/ $/$ /day. The multiplication per generation (Ro) ranged from 7.738 to 24.064 times on the above mentioned temperatures, respectively. Results in Table 4, 5 \& 6 showed that, feeding capacity of $T$. swirskii was greatly influenced by food types and temperature. Tested tetranychoid mites (E. orientalis, C. pulcher, P. ulmi and T. urticae) were more favorable as prey than tested insects (the whitefly B. tabaci, the scale insects $C$. ficus \& $C$. hesperidium and the aphid, A. duranta). The female predator immatures and adult consumed prey immatures $21.3 \& 148.0$ of E. orientalis and $10.3 \& 120.7$ of $T$. urticae, (Table 4); compared to $8.8 \& 112.32^{\text {nd }}$ instar nymphs of B. tabaci and $3.7 \&$ 70.0 A. duranta adults, Table (5).

Although the size of E. orientalis adults are larger than those of $C$. pulcher and immatures of $T$. urticae and nearly equal to $P$. ulmi adults, yet the devoured daily rate during predator immatures and adult stage of the citrus brown mite was greater. This phenomenon appeared in prey insects, as inspite of the large size of whitefly $2^{\text {nd }}$ instar nymphs than those of the two scale insects, yet the attacked daily rate of the former was greater. Concerning predator male, it followed similar trend in consumption of prey species and stages (Tables $4 \& 5)$.

Data presented in table (6) revealed that temperature significantly affected amount of consumed prey. When $T$. swirskii fed on $E$. orientalis immatures, the total amount of consumed prey individuals/ predator different develop-mental 
stages and sex increased as temperature increased from 15 to $35^{\circ} \mathrm{C}$. These increments sharply jumped between 20 and $25^{\circ} \mathrm{C}$. The total individuals attacked/predator immatures and adult was in an ascending order $5.0 \& 23.7 ; 21.3 \& 148.0 ; 28.3 \&$ $176.5 ; 34.0 \& 185.3$ and $37.3 \& 194.5$ individuals at $15,25,30,32$, and $35^{\circ} \mathrm{C}$, respectively. Thus, high temperature from 30 to $35^{\circ} \mathrm{C}$ were more suitable for prey consumption. This was assured by obtained results in the life table parameters of the predator as (rm) was, $0.227,0.269$ and 0.289 at 30,32 and $35^{\circ} \mathrm{C}$, respectively.

Thus, the present results revealed, that $E$. orientalis immatures and date palm pollens were the preferable food and high temperature degrees from 30 to $35^{\circ} \mathrm{C}$ were the most suitable, for mass rearing T. swirskii.

\section{REFERENCES}

Abou-setta, M. A.; Sorrell, R. W. and Childers, C. C., 1986. Life 48: A basic computer program to calculate life table parameters for an insect or mite species. Fla. Entomol., 69: 690-697.

Ali, Fatma, S., 1998. Life table of Phytoseiulus macropilis (Banks) (Gamasida: Phytoseiidae) at different temperatures. Exp. Appl. Acarology, 22: 335-342.

Caceres, S. and Childers, C. C., 1991. Biology and life tables of Galendromus helveolus (Chant) (Acari: Phytoseiidae), on Florida citrus. Envirom. Entomol., 20 (1): 224-228.

Decou, G. C., 1994. Biological control of the twospotted spider mite (Acarina: Tetranychidae), on commercial strawberries in Florida with Phytoseiulus persimilis (Acarina: Phytoseiidae). Florida Entomologist. 77 (1): 33-41 pp.

El-Laithy, A. Y. M., 1992. Some aspects on the use of the predatory mite Phytoseiulus persimilis Athias-Henriot for biological control of the two spotted spider mite Tetranychus urticae

Koch in greenhouses in Egypt. J. Plant. Dis. Prot. 99 (1): 93-100.

Heikal, I. H. and Ibrahim, G. A., 2001. Release of Phytoseiulus macropilis (Banks) to control Tetranychus urticae Koch on strawberry in
Ismailia governorate, Egypt (Acari: Phytoseiidae, Tetrany-chidae). Egypt. J. Agric. Res., 79 (3): 893-906.

Heikal, I. H. and Mowafi, M. H., 1998. Biological control of Tetranychus urticae on bean plants by two introduced predators. Al-Azhar J. Agric. Res., 27: 185-196.

Heikal, I. H.; Fawzy, M. M., Ibrahim, H. M. and Ibrahim, G. A., 2000. Preliminary studies on the release of the predatory mite Phytoseiulus macropilis (Banks) on strawberry plant to control Tetranychus urticae Koch (Acari: Tetranychidae, Phytoseiidae). Egypt. J. Agric. Res., 78 (4): 1517-1523.

Remos, M. and Redriguez, H., 1995. Efficiency of control of Tetranychus tumidus (Banks) by Pytoseiulus macropilis (Banks) in banana greenhouse trials. Revista de protection vegetal, 10: 207-211.

van de Vrie, M. and Boorsma, A., 1970. The influence of the predaceous mite Typhlodromus (A.) potentillae (Garman) on the development of Panonychus ulmi (Koch) on apple grown under various nitrogen conditions. Entomophaga, 15: 291-304.

van de Vrie, M. and Kropczynsha, 1965. The influence of predatory mites on the population development of Panonychus ulmi (Koch) on apple. Proc. $5^{\text {th }}$ European Symp. Acarology, Milano 1965, Bull, Zool. Agr. Bachic., Series II, 7: 119-120.

Walter, D. E., 1992. Leaf surface structure and the distribution of phytoseiid mites (Acarina: Phytoseiidae) in South Eastern Australian Forests. Aust. J. Zool., 40: 593-603.

Watanabe, M. A.; De Morales, G. J.; Gastaldo, I. J. R. and Nicolella, G., 1994. Biological control of two-spotted mite with predatory phytoseiids (Acari: Tetranychidae, Phytoseiidae) on cucumber and strawberry. Scientia, Agricola, 51 (1): 75-81.

Zaher, M. A., 1986. survey and ecological studies on phytophagous, predaceous and soil mites in Egypt. II-A: Predaceous and nonphytophagous mites. PL. 480 Programme U.S.A. Project No. 139 (Report). 